Feb;20(2):247-253), and language regression occurs in 43\% of the patients with sleeppotentiated epileptic activity in the EEG. (Sanchez-Fernandez I. et al. Neurology 2012 May 29;78(22):1721-1727). The present article links acquired epileptic aphasia with sleep-potentiated epileptic activity in the EEG and MRI findings of early thalamic injury. The thalamus has a role not only in the facilitation of seizure activity during non-REM sleep but also in sleep-related electrical status epilepticus and associated language and behavioral regression.

\title{
THERAPY OF ENCEPHALOPATHY WITH ESES/CSWS
}

Researchers at University of Pavia, Italy review treatment options for electrical status epilepticus in sleep (ESES)/continuous spikes and waves during slow sleep (CSWS) and their comparative value. ESES/CSWS is an age-related, self-limiting disorder characterized by epilepsy with various seizure types, neuropsychological regression, and a typical EEG pattern of continuous epileptiform activity for more than $85 \%$ of non-rapid eye movement (non-REM) sleep. ESES/CSWS is rare, accounting for $0.2-0.5 \%$ of childhood epilepsies. The goal of treatment is not only to control clinical seizures but also to improve neuropsychological functions and prevent potential cognitive deterioration. When the EEG pattern of ESES is refractory to AEDs, more aggressive therapeutic approaches are considered, but evidence of efficacy is dependent on uncontrolled studies and case reports. The authors' suggested approach to treatment begins with the AEDs valproic acid, benzodiazepines, or levetiracetam. If unresponsive after 6 months, the addition of steroids is applicable. Phenobarbital and carbamazepine should be avoided since these AEDs increase synchronous bilateral epileptic discharges in the EEG and worsen clinical manifestations. (Veggiotti P, Pera MC, Teutonico F, Brazzo D, Balottin U, Tassinari CA. Therapy of encephalopathy with status epilepticus during sleep (ESES/CSWS syndrome): an update. Epileptic Disord 2012 Mar;14(1):111). (Respond: Dr P Veggiotti, Instituto Neurologico C Mondino, Via Mondino 2, 27/100 Pavia, Italy. E-mail: pveggiot@unipv.it).

COMMENT. In this report, ESES and CSWS are considered synonymous but this opinion is sometimes debated. Cognitive impairment is a hallmark of the syndrome, either global or selective, involving language regression as in acquired epileptic aphasia (Roulet PE, Davidoff V, Despland PA, Deonna T. Dev Med Child Neurol 1993 Aug;35(8):661-674). The pathophysiological mechanisms of CSWS and associated neuropsychological deficits are poorly understood.

Investigators in Kiel, Germany compared the results of functional MRI in 12 children with CSWS to electric source analysis. Spike-related positive activations and negative deactivations involved bilateral perisylvian region and cingulate gyrus in all cases, frontal cortex in 5, parietal cortex in 1, and thalamus in 5 cases. Despite etiological heterogeneity, patients with CSWS were characterized by activation of the similar neuronal network: perisylvian region, insula and cingulate gyrus. The influence of epileptic spikes on neuronal networks important in the consolidation of memory traces during sleep may explain the association of CSWS with neuropsychological deficits. (Siniatchkin M, Groening K, Moehring J, et al. Neuronal networks in children with continuous spikes and waves during slow sleep. Brain 2010 Sep;133(9):2798-2813). 